\section{P165 TB-ST RAPID TEST FOR TUBERCULOSIS DIAGNOSIS IN A RESOURCE POOR SETTING: ARE CASES OF TUBERCULOSIS GOING UNDIAGNOSED?}

doi:10.1136/thx.2010.151043.16

${ }^{1} \mathrm{~N}$ Javanshir, ${ }^{1} \mathrm{D}$ S Shah, ${ }^{1} \mathrm{D}$ E Jennings, ${ }^{2} \mathrm{~J}$ Ivanyi, ${ }^{3} \mathrm{H}$ J Milburn. ${ }^{1} \mathrm{GKT}$ School of Medicine, King's College, London, UK; ' $C$ Clinical and Diagnostic Sciences, King's College, London, UK; ${ }^{3}$ Dept Respiratory Medicine, Guy's and St Thomas' NHS Foundation Trust, London, UK

Introduction Diagnosis of tuberculosis (TB) ideally involves culture and sensitivity of the organism but in low income countries this is not practised routinely. The World Health Organisation estimates that substandard detection occurs in $40 \%$ of patients globally, with many diagnosed on clinical suspicion or response to medication. TB produces a strong antibody response suitable for simple, inexpensive and rapid serodiagnostic assays. Ongoing evaluation of a new point of care rapid serological test based on lateral flow immunochromatography (TB-ST Rapid Test, Lionex, Germany) has shown 100\% specificity, with no false positive tests in normal controls and latently infected patients, but sensitivity of $36 \%$, with false negatives in culture proven TB.

Aim Using this test in a resource poor setting to investigate whether cases of active TB may be being missed by current diagnostic methods. Methods 498 patients in chest and HIV clinics in two rural Kenyan hospitals were investigated with the TB-ST Rapid Test and a scored questionnaire to determine symptoms and risk of $\mathrm{TB}$. Results were compared with clinical diagnoses made, usually based on symptoms alone. Chest radiographs were performed in only 111 and sputum smears in 75 .

Results $127 / 498$ patients were HIV positive. Of these, only $59(46 \%)$ had a clinical diagnosis of $\mathrm{TB}$, whereas $87(68.5 \%)$ had significant $\mathrm{TB}$ symptoms and/or risk factors, and $82(64.6 \%)$ tested TB-ST positive $(p<0.001)$. Therefore clinical diagnosis accounted for significantly fewer diagnoses of active TB than suggested by either symptom and risk score or TB-ST rapid results in the HIV+ population. Of the 375 HIV - patients, 73 (19.7\%) had a clinical diagnosis of TB, $46(12.3 \%)$ scored positive for TB on the questionnaire, and 149 (40.2\%) were TB-ST $+(p<0.001)$. Abstract P165 Table 1 shows positive and negative TB-ST results related to positive and negative sputum smears in $34 \mathrm{HIV}+$ and $41 \mathrm{HIV}$ - patients.

Abstract P165 Table 1

\begin{tabular}{lllcc}
\hline & $\begin{array}{l}\text { HIV +ve } \\
\text { TB-ST +ve }\end{array}$ & $\begin{array}{l}\text { HIV +ve } \\
\text { TB-ST - ve }\end{array}$ & $\begin{array}{l}\text { HIV - ve } \\
\text { TB-ST +ve }\end{array}$ & $\begin{array}{l}\text { HIV - ve } \\
\text { TB-ST - ve }\end{array}$ \\
\hline Smear negative (\%) & $13(38)$ & $8(23.5)$ & $9(21.9)$ & $11(26.8)$ \\
Smear positive (\%) & $8(23.5)$ & $5(15)$ & $17(41.5)$ & $4(9.8)$ \\
\hline
\end{tabular}

Conclusions Many more patients had positive TB-ST and risk and symptom scores than were being diagnosed with active $T B$, suggesting that TB may not always be being diagnosed or treated. Sputum smears were in greater agreement with the TB-ST in HIVbut not HIV + patients, in whom there were considerably fewer positive smears than TB-ST results.

\section{P166 QUANTIFERON TESTING IN CLOSE CONTACTS OF SMEAR POSITIVE PULMONARY TB IDENTIFIES PEOPLE AT LOW RISK OF SECONDARY PROGRESSION}

doi:10.1136/thx.2010.151043.17

P Haldar, H Thuraisingam, H Patel, W Hoskyns, G Woltmann. University Hospitals of Leicester, Leicester, UK

Introduction Interferon gamma release assays (IGRAs) are recommended for screening close contacts of patients with active tuber- culosis (TB) in the UK. Compared with tuberculin skin testing, greater specificity with IGRAs reliably informs the need for chemoprophylaxis in screened contacts by identifying latent Mycobacterium tuberculosis infection (LTBI) associated with a high risk of secondary disease progression. However, the risk of secondary progression varies with the nature of disease in the primary case and is greatest for contacts of smear positive pulmonary disease. The role of IGRAs in this very high risk population is not clear.

Aim To evaluate the benefit of screening with QuantiFERON ${ }^{\circledR}-\mathrm{TB}$ Gold (OFT) in contacts of smear positive pulmonary TB.

Methods A prospective observational study. We have offered OFT based single step screening programme for all close contacts of smear positive pulmonary TB since January 2007. We present 2-year followup data in tested and untested contacts that did not receive chemoprophylaxis. Secondary disease risk is estimated with Kaplan-Meier analysis and subgroups compared with the log-rank test.

Results 576 recorded contacts for 92 smear positive cases. Median follow-up 693 days (range 287-1146 days). 467 (81.1\%) of contacts were OFT screened. $36 \%$ of tested contacts had a positive OFT; 83 OFT positive contacts did not receive chemoprophylaxis (group A); 101 contacts were not screened and untreated (group B); 301 were OFT negative (group C). Secondary disease occurred in 22 contacts (11 group A, 5 group B). Progression rates at 12 and 24 months are shown (Abstract P166 Table 1). Compared with the untested subgroup, a positive OFT results did not significantly increase progression risk (RR 2.1 ( $95 \%$ CI 0.7 to 5.9), $\mathrm{p}=0.13$ ). A negative OFT result did significantly lower progression risk (RR 0.28 (95\% CI 0.09 to 0.82 ) at 2 years, $\mathrm{p}=0.01$ ). The negative predictive value of OFT compared with not testing was $93.2 \%$ (95\% CI 85.8 to 97.5 ).

\section{Abstract P166 Table 1}

\begin{tabular}{llll}
\hline \multirow{2}{*}{ Observation period/days } & \multicolumn{4}{l}{ Progression risk \% (SEM) } \\
\cline { 2 - 4 } & Group A & Group B & Group C \\
\hline $0-365$ & $11.7(4.0)$ & $5.7(2.0)$ & $1.0(1.0)$ \\
$365-730$ & $15.2(4.0)$ & $7.4(3.0)$ & $2.0(1.0)$ \\
\hline
\end{tabular}

Conclusion In contrast with other TB disease types, OFT screening in contacts of smear positive pulmonary $\mathrm{TB}$ does not identify contacts for chemoprophylaxis, but rather identifies contacts that we may choose not to treat.

\section{P167 CHARACTERISATION OF TUBERCULOUS MEDIASTINAL LYMPHADENOPATHY ON CT AND CORRELATION WITH BIOMARKERS AND CHEST RADIOGRAPH FINDINGS}

doi:10.1136/thx.2010.151043.18

A Singanayagam, P George, D Connell, S Kaneria, J Dhariwal, P Molyneaux, M Wickremasinghe, A Lalvani, A Wright, O M Kon. St Mary's Hospital, London, UK

Introduction The aim of this study was to characterise the anatomical pattern of mediastinal lymphadenopathy on CT in active tuberculosis (TB) and assess correlation with biomarkers and chest radiograph (CXR) findings.

Methods We conducted a retrospective analysis of patients treated for active TB with mediastinal lymphadenopathy at a tertiary centre between January 2007 and May 2009. CT scans performed prior to commencement of therapy were reviewed by two radiologists to assess for pathologically enlarged lymph nodes (LN) (short axis diameter $>1.0 \mathrm{~cm}$ ) at each of the $\mathrm{LN}$ stations defined by the International Association for the Study of Lung Cancer (IASLC).

Results 55 patients were included in the study. $56.3 \%$ of enlarged LNs were right sided, $32.9 \%$ were in central LN groups and $10.8 \%$ were left sided. $40.0 \%$ of patients had evidence of necrosis within LNs. The most frequently occurring enlarged LNs were in the 